\title{
Relationships between Dietary Habits and Allostatic Load Index in Metabolic Syndrome Patients
}

\section{Ju Young Kim*}

Department of Family Medicine, Seoul National University Bundang Hospital, Seoul National University College of Medicine, Seongnam, Korea

Background: Allostatic load (AL) measures overall physiological wear and tear on one's body, as a preclinical marker of pathophysiologic processes that precede the onset of disease. We studied the association of dietary habits with AL.

Methods: Consecutive patients visiting a tertiary hospital Health Promotion Center from September 2009 to February 2010 , older than 20 years with metabolic syndrome were selected for study $(n=204)$. By multivariable linear regression analysis, we investigated the association of various dietary habits evaluated by questionnaires.

Results: In male, multivariable linear regression showed a significant negative association between fat preference and $\mathrm{AL}$ with $\mathrm{BMI} \geq 30$ (1st quartile [Q] vs. 2Q: $\beta=-3.71$; $95 \%$ confidence interval $[\mathrm{CI}],-6.26$ to -1.16 ), a significant negative association between salt preference and AL with BMI 25-30 ( $\beta=-1.36$; 95\% CI, -2.46 to -0.26 ), a negative association between appetite control and AL with BMI < 25 (1Q vs. 3Q: $\beta=-1.54 ; 95 \%$ CI, -3.00 to -0.096 ), a significant positive association between appetite control and AL with BMI 25-30 (1Q vs. 3Q: $\beta=1.30 ; 95 \%$ CI, 0.12 to 2.48), and a significant positive association between eating in response to food cues and AL in males with BMI 25-30 (1Q vs. 4Q: $\beta=1.09 ; 95 \%$ CI, 0.020 to 2.15$)$.

Conclusion: Our results suggest that metabolic syndrome patients should be discouraged from eating fat and eating in response to food cues, and should be educated about nutrition and balanced diet.

Keywords: Allostasis; Metabolic Syndrome; Food Habits; Food Preferences

\section{INTRODUCTION}

Allostatic load (AL), a concept introduced by McEwen and Stellar, ${ }^{1)}$ measures overall physiological wear and tear on one's

Received: August 31, 2012, Accepted: September 3, 2013

${ }^{*}$ Corresponding Author: Ju Young Kim

Tel: +82-31-787-7796, Fax: +82-31-787-4078

E-mail: kabbey@hanmail.net

Korean Journal of Family Medicine

Copyright (C) 2013 The Korean Academy of Family Medicine

() This is an open-access article distributed under the terms of the Creative Commons Attribution Non-Commercial License (http://creativecommons.org/licenses/by-nc/3.0) which permits unrestricted noncommercial use, distribution, and reproduction in any medium, provided the original work is properly cited. body, as a preclinical marker of pathophysiologic processes that precedes the onset of disease. The basic premise is that adaptation to the demands of everyday living requires the body to react physiologically to a wide variety of stressors, a dynamic process referred to as allostasis.

Previous studies support the hypothesis that higher AL is associated with poorer health status. An initial application of AL using measures of the hypothalamic-pituitary-adrenal (HPA) axis, cardiovascular activity, adipose tissue deposition, and glucose metabolism was developed by Seeman et al. ${ }^{2)} \mathrm{AL}$ prospectively predicted clinically relevant outcomes including incident cardiovascular events, physical function, cognitive decline, and mortality. ${ }^{3,4)}$ These findings provide evidence that $\mathrm{AL}$ captures physiological changes that precede the occurrence 
of clinical disease and, hence, represent a meaningful step in the disease development process. As a result, consideration of the relationship between an exposure of interest and $\mathrm{AL}$ may give some insight into the mechanisms by which the exposure may ultimately influence specific health outcomes. Beyond the basic physiological point of view, conceptual elaboration of AL and its relationship to life experiences has been provided by many studies. $^{5,6)}$

The role of dietary habits as a major contributor to chronic conditions has been well established. Unhealthy dietary patterns, such as high intake of refined grains, meats, and sweets and soft drinks, have been associated with obesity ${ }^{7)}$ and higher risk of all-cause, cancer, and cardiovascular disease (CVD) mortality. ${ }^{8)}$ Environmental stressors, including diet, have been proposed as contributors to $\mathrm{AL}{ }^{9)} \mathrm{AL}$ may be triggered as a response to continued energy storage above a person's needs, which is heavily influenced by food choices and behaviors. ${ }^{10)}$ Yet, only one study investigated the effect of dietary patterns on cumulative dysregulation in which meat, processed meat, and French fry dietary patterns were shown to be associated with high AL. ${ }^{11)}$

$\mathrm{AL}$ has been associated with increased risk of incident CVD and mortality, and declines in cognitive and physical function, and these results have been shown to be stronger than those observed for metabolic syndrome. ${ }^{4)}$ Furthermore, it has been shown that higher scores of AL were associated with increased odds of abdominal obesity, type 2 diabetes, CVD, hypertension, and arthritis; most of these associations were also stronger than those observed with metabolic syndrome. ${ }^{12)}$ Although metabolic syndrome patients are more susceptible to chronic diseases, there has been no study on the association of behavioral factors in this population to AL. Our study investigated the association of dietary habits with $\mathrm{AL}$ in metabolic syndrome patients, in order to determine which specific dietary habits are risks to AL in metabolic syndrome patients.

\section{METHODS}

\section{Subjects}

Consecutive patients visiting Seoul National University Bundang Hospital Health Promotion Center from September 2009 to February 2010, both men and women older than 20 years with metabolic syndrome, were selected for study. The National Cholesterol Education Program's Adult Treatment Panel III definition for metabolic syndrome was implemented, defined as meeting three of the five following criteria: 1) waist circumference $\geq 90 \mathrm{~cm}$ (men), waist circumference $\geq 80 \mathrm{~cm}$ (women); 2) fasting serum glucose $\geq 110 \mathrm{mg} / \mathrm{dL} ; 3)$ serum triglyceride $\geq 150 \mathrm{mg}$ / $\mathrm{dL} ; 4)$ serum high density lipoprotein (HDL) cholesterol $<40$ $\mathrm{mg} / \mathrm{dL}$ (men), HDL cholesterol $<50 \mathrm{mg} / \mathrm{dL}$ (women); 5) blood pressure $\geq 130 / 85 \mathrm{~mm} \mathrm{Hg}$. Among a total of 292 participants, 88 samples with missing values on variables for calculation of $A L$ index were excluded. The final 204 participants were included in analysis.

\section{Allostatic Load Index}

Operationalization of $\mathrm{AL}$ was developed through an algorithm based on ten biomarkers: ${ }^{4)}$ systolic and diastolic blood pressure reflecting cardiovascular activity; waist/hip ratio reflecting metabolism and adipose tissue deposition; HDL and total cholesterol reflecting cardiovascular health; glycosylated hemoglobin reflecting glucose metabolism; cortisol excretion reflecting HPA axis activity; serum dihydroepiandrosterone sulfate also reflecting HPA axis activity; and norepinephrine and epinephrine reflecting sympathetic nervous system activity. Each of the parameters was classified into quartiles, and AL was measured by summing the number of parameters for which the subject fell into the highest-risk quartile.

Previous studies used various biomarkers for AL since there is no single formula to obtain AL index. ${ }^{13)}$ Some studies obtained AL index without biomarkers to reflect the HPA axis. ${ }^{14)}$

In our study we implemented twelve parameters: systolic blood pressure, diastolic blood pressure, waist circumference, body fat percentage measured by impedance, serum low density lipoprotein (LDL) cholesterol, serum triglyceride, serum HDL cholesterol, fasting serum glucose, serum hemoglobin alc, serum insulin, serum interleukin-6, and serum C-reactive protein. Final $\mathrm{AL}$ index was measured by summing the number of parameters pertaining to the high quartile.

\section{Measures of Dietary Habits}

A non-validated Korean-based comprehensive survey was developed on dietary habits consisting of seven categories (Table 1): 1) meal time regularity (regularity), 2) dietary preference- 
Table 1. Survey questions on dietary habits by category

\begin{tabular}{|c|c|}
\hline Categories of dietary habits & Contents of questionnaires \\
\hline \multirow[t]{4}{*}{ Meal time regularity } & Meals are often skipped due to busy schedule. \\
\hline & Engage in generally irregular lifestyle, including meals. \\
\hline & Breakfast and lunch are small whereas dinner is usually large. \\
\hline & Engage in frequent bedtime snacks. \\
\hline \multirow[t]{2}{*}{ Dietary preference (carbohydrates) } & Snacks usually include sweet food, such as bread, cookies, coffee, soda, juice, and fruit. \\
\hline & There are often junk food, such as chips and cookies, within reach. \\
\hline \multirow[t]{2}{*}{ Dietary preference (fat) } & Enjoy dietary oily food, such as Chinese or Western food. \\
\hline & Enjoy dietary fried food. \\
\hline \multirow[t]{13}{*}{ Dietary preference (salt) } & Enjoy strong-tasting food. \\
\hline & Prefer kimchi instead of fresh vegetable. \\
\hline & Enjoy cooked or seasoned rice. \\
\hline & Prefer Chinese or Japanese food over Western food. \\
\hline & Enjoy dried fish. \\
\hline & Salted fish are missed when absent in a meal. \\
\hline & Often add seasonings to served food. \\
\hline & Often eat the entire soup or stew. \\
\hline & Fried food or raw fish is usually deeply dipped into soy sauce. \\
\hline & Often eat outside or delivered food. \\
\hline & Often add mayonnaise or dressings to food. \\
\hline & Usually do not eat the soup portion of ramen. \\
\hline & Usually do not eat salted fish or pickled food. \\
\hline \multirow[t]{4}{*}{ Knowledge in nutrition and balanced diet } & Not aware of which food are nutritious. \\
\hline & Foods like tofu, beans, egg, fish, or lean meat are not preferred. \\
\hline & Usually picky on what to eat. \\
\hline & Not aware of the appropriate amount of calories. \\
\hline \multirow[t]{4}{*}{ Appetite control } & Often eat dessert despite being full. \\
\hline & Often eat whenever other family members eat. \\
\hline & Often eat whenever there is food. \\
\hline & Often regret for having eaten too much. \\
\hline \multirow[t]{10}{*}{ Eating to food cues } & The refrigerator is always filled with some snack. \\
\hline & Often relieve stress by dietary. \\
\hline & Do not spare left-over food, feeling guilty. \\
\hline & During holidays always spend time indoors doing nothing lying down. \\
\hline & Have gone on diets many times. \\
\hline & Eat outside at least three times a week. \\
\hline & Often eat fast-food. \\
\hline & Usually eat fast. \\
\hline & Often swallow without having chewed food properly. \\
\hline & Always experience weight gain after holidays or vacations. \\
\hline
\end{tabular}


carbohydrates (CHO preference), 3) dietary preference-fat (fat preference), 4) dietary preference-salt (salt preference), 5) knowledge of nutrition and balanced diet (knowledge \& balance), 6) appetite control (appetite control), and 7) eating in response to food cues (eating to food cues). The survey consisted of a total of 39 questions, for which the answer choices were 1) not at all, 2) usually not, 3) sometimes yes, and 4) always yes. Answer choices were gathered as numeric values accordingly as $1,2,3$, or 4 . They were then averaged for each category, subsequently categorized in quartiles for analysis.

\section{Covariates}

Age and body mass index (BMI) were included as continuous variables. Sex, self-reported current medication for diabetes, dyslipidemia, and hypertension were included as binary variables. Smoking was categorized as never smokers, former smokers, and current smokers. Alcohol consumption was categorized as drinking on average 0 gram per day, less than 10 grams per day, and more than 10 grams per day. The regular exercise group was defined by exercise frequency more than 3 times per week.

\section{Statistical Analysis}

Multivariable linear regression analysis was used for the main analysis to examine associations between $\mathrm{AL}$ and dietary habits. In order to evaluate an overall trend in the relationship between questionnaire-based dietary habits and AL, cubic spline analysis was performed with restricted cubic spline smoothing. We also performed multivariable logistic regression to find the odds ratio between each parameter of AL index and selected dietary habits. All results with a P-value of $<0.05$ were considered statistically significant. Stata ver. 12.0 (Stata Co., College Station, TX, USA) was used for all analyses.

\section{RESULTS}

\section{Study Population Characteristics}

Basic characteristics of our study population are shown in Table 2. The total number of people in the study population was 204 and there were 146 males (71.4\%). The mean age for male subjects was 48.8 , whereas the mean age for female subjects was 54.2 .

\section{Association of Dietary Habits to Allostatic Load Index}

Multivariable linear regression adjusted for age, BMI, alcohol consumption, smoking, medication to diabetes, medication to hypertension, medication to dyslipidemia, showed a significant negative association between fat preference and AL in males with $\mathrm{BMI} \geq 30$ (1st quartile $[\mathrm{Q}]$ vs. $2 \mathrm{Q}: \beta=-3.71 ; 95 \%$ confidence interval $[\mathrm{CI}],-6.26$ to -1.16$)$, a significant negative association between salt preference and AL in males with BMI 25-30 (1Qvs. $4 \mathrm{Q}: \beta=-1.36$; $95 \% \mathrm{CI},-2.46$ to -0.26 ), a negative association between appetite control and $\mathrm{AL}$ in males with $\mathrm{BMI}$ $<25$ (1Qvs. 2Q: $\beta=-1.06$; 95\% CI, -2.09 to -0.037 ) (1Qvs. 3Q: $\beta=-1.54 ; 95 \% \mathrm{CI},-3.00$ to -0.096$)$, a significant positive association between appetite control and AL in males with BMI 25-30 (1Qvs. 3Q: $\beta=1.30$; $95 \%$ CI, 0.12 to 2.48 ), and a significant positive association between eating in response to food cues and $\mathrm{AL}$ in males with BMI 25-30 (1Qvs. 4Q: $\beta=1.09 ; 95 \%$ CI, 0.020 to 2.15 ) as shown in Table 3.

In contrast to male subjects, female subjects were stratified into normal weight and overweight/obese subjects due to the low number of total female subjects. As shown in Table 4, most observations were shown to be statistically not significant except for dietary behavior, for which multivariable linear regression showed a positive association with $\mathrm{AL}$ (1Qvs. 3Q: $\beta=2.19 ; 95 \%$ CI, 0.67 to 3.71 ).

\section{Association between Highest Quartile of Dietary Habits and Parameters of Allostatic Load}

Age adjusted logistic regression analysis was performed between highest quartile of dietary preference for fat, dietary preference for salt, and appetite control, and all twelve parameters of allostatic index included in this study (Table 5). In both males and females, dietary preference for fat was significantly associated with higher body fat percentage (males: adjusted odds ratio [aOR], 1.16; 95\% CI, 1.02 to 1.32) (females: aOR, 1.32; 95\% $\mathrm{CI}, 1.10$ to 1.59$)$. In male subjects, dietary preference for salt was significantly associated with higher LDL (aOR, 1.02; 95\% CI, 1.00 to 1.04$)$. In male subjects, appetite control was associated to higher LDL (aOR, 1.02; 95\% CI, 1.00 to 1.04), and insulin (aOR 1.02 ; $95 \% \mathrm{CI}, 1.01$ to 1.04$)$. In females, appetite control was associated to higher body fat percentage (aOR, 1.18; 95\% CI, 1.02 to 1.36 ), and higher LDL (aOR, 1.01; 95\% CI, 1.01 to 1.10). 
Table 2. Basic characteristics of study population $(n=204)$

\begin{tabular}{|c|c|c|c|}
\hline Characteristic & Male $(\mathrm{n}=146)$ & Female $(n=58)$ & P-value \\
\hline Age (y) & $48.8 \pm 9.7$ & $54.2 \pm 8.2$ & $<0.01$ \\
\hline Body mass index $\left(\mathrm{kg} / \mathrm{m}^{2}\right)$ & $26.6 \pm 2.8$ & $25.8 \pm 3.7$ & 0.069 \\
\hline$<25$ & $35(24.0)$ & $26(44.8)$ & \\
\hline $25-30$ & $94(64.4)$ & $24(41.4)$ & \\
\hline$\geq 30$ & 17 (11.6) & $8(13.8)$ & \\
\hline Smoking & & & $<0.01$ \\
\hline Never & $20(13.7)$ & $53(91.4)$ & \\
\hline Former & $75(51.4)$ & $1(1.7)$ & \\
\hline Current & $51(34.9)$ & $4(6.9)$ & \\
\hline Alcohol intake & & & $<0.01$ \\
\hline None & $7(5.1)$ & $19(44.2)$ & \\
\hline Moderate drinking & $82(60.3)$ & $22(51.2)$ & \\
\hline Problem drinking & 47 (34.6) & $2(4.7)$ & \\
\hline Regular exercise & & & 0.081 \\
\hline$\geq 3 /$ wk & $67(45.9)$ & $18(31.6)$ & \\
\hline Hypertension medication & & & 0.726 \\
\hline No & $104(73.2)$ & $40(70.2)$ & \\
\hline Yes & $38(26.8)$ & $17(29.8)$ & \\
\hline Diabetes mellitus medication & & & 0.072 \\
\hline No & $128(90.1)$ & $56(98.2)$ & \\
\hline Yes & $14(9.9)$ & $1(1.8)$ & \\
\hline Dyslipidemia medication & & & 0.390 \\
\hline No & $122(85.9)$ & $46(80.7)$ & \\
\hline Yes & $20(14.1)$ & $11(19.3)$ & \\
\hline Systolic blood pressure (mm Hg) & $127.5 \pm 13.6$ & $127.8 \pm 13.9$ & 0.857 \\
\hline Diastolic blood pressure (mm Hg) & $80.9 \pm 10.8$ & $76.6 \pm 8.6$ & $<0.01$ \\
\hline Waist circumference $(\mathrm{cm})$ & $93.4 \pm 6.8$ & $88.5 \pm 7.5$ & $<0.01$ \\
\hline Body fat (\%) & $25.7 \pm 3.5$ & $33.4 \pm 3.9$ & $<0.01$ \\
\hline C-reactive protein (mg/dL) & $0.2 \pm 0.3$ & $0.2 \pm 0.2$ & 0.896 \\
\hline Interleukin-6 (IU/mL) & $1.2 \pm 2.3$ & $1.1 \pm 1.8$ & 0.666 \\
\hline Triglyceride & $174.4 \pm 73.4$ & $154 \pm 56.4$ & 0.070 \\
\hline Low density lipoprotein cholesterol & $112.4 \pm 26.7$ & $118.0 \pm 29.4$ & 0.030 \\
\hline High density lipoprotein cholesterol & $43.9 \pm 9.3$ & $47.9 \pm 9.6$ & $<0.01$ \\
\hline Glucose & $104.5 \pm 21.0$ & $97.4 \pm 17.6$ & 0.024 \\
\hline Hemogloblin Alc (\%) & $6.0 \pm 0.9$ & $5.9 \pm 0.6$ & 0.483 \\
\hline Insulin & $21.5 \pm 28.9$ & $18.6 \pm 21.9$ & 0.489 \\
\hline
\end{tabular}

Values are presented as mean \pm SD or number (\%). P-value was calculated using t-test or chi-square test. 
Table 3. Association between allostatic load index and dietary habits in male subjects (total $\mathrm{n}=146$ )

\begin{tabular}{|c|c|c|c|c|c|c|}
\hline \multirow{2}{*}{ Variable } & \multicolumn{2}{|c|}{$\mathrm{BMI}<25$} & \multicolumn{2}{|c|}{$25 \leq \mathrm{BMI}<30$} & \multicolumn{2}{|c|}{$\mathrm{BMI} \geq 30$} \\
\hline & Univariable & Multivariable & Univariable & Multivariable & Univariable & Multivariable \\
\hline \multicolumn{7}{|l|}{ Regularity } \\
\hline 1stQuartile (4-7) & 1 & 1 & 1 & 1 & 1 & 1 \\
\hline 2nd Quartile (8-9) & -0.18 ( -1.53 to 1.18$)$ & 0.026 (-1.33 to 1.38) & $0.52(-0.43$ to 1.48$)$ & $0.62(-0.33$ to 1.58$)$ & $-0.50(-4.64$ to 3.64$)$ & 2.64 (-3.78 to 9.05$)$ \\
\hline 3rdQuartile (10-11) & 0.53 (-0.64 to 1.71$)$ & $-0.01(-1.24$ to 1.22$)$ & 0.19 (-0.60 to 0.97$)$ & 0.46 (-0.35 to 1.26$)$ & 0.83 (-2.38 to 4.04$)$ & $0.68(-3.15$ to 4.50$)$ \\
\hline 4th Quartile (12-16) & -0.69 (-2.10 to 0.72$)$ & $-1.05(-2.49$ to 0.40$)$ & $0.60(-0.48$ to 1.69$)$ & $0.77(-0.38$ to 1.91$)$ & 0.67 (-2.36 to 3.69$)$ & $1.47(-2.45$ to 5.38$)$ \\
\hline \multicolumn{7}{|c|}{ Carbohydrates preference } \\
\hline 1stQuartile (2-3) & 1 & 1 & 1 & 1 & 1 & 1 \\
\hline 2nd Quartile (4) & -0.37 (-1.55 to 0.81$)$ & $-0.13(-1.42$ to 1.16$)$ & 0.04 (-0.85 to 0.93$)$ & 0.26 (-0.60 to 1.13$)$ & 0.33 (-2.70 to 3.37$)$ & $-0.022(-3.69$ to 3.65$)$ \\
\hline 3rd Quartile (5) & -0.99 (-2.23 to 0.25$)$ & $-0.75(-2.07$ to 0.58$)$ & 0.21 (-0.62 to 1.03$)$ & 0.36 (-0.45 to 1.16$)$ & $-0.79(-3.83$ to 2.24$)$ & $-0.43(-4.76$ to 3.89$)$ \\
\hline 4th Quartile (6-8) & -0.24 (-1.88 to 1.39) & 0.44 (-1.34 to 2.22$)$ & 0.39 (-0.85 to 1.63$)$ & 0.56 (-0.68 to 1.81$)$ & 0.33 (-4.84 to 5.51$)$ & 2.59 (-3.53 to 8.71$)$ \\
\hline \multicolumn{7}{|l|}{ Fatpreference } \\
\hline 1stQuartile (2-3) & 1 & 1 & 1 & 1 & 1 & 1 \\
\hline 2nd Quartile (4-5) & 0.04 (-1.11 to 1.20$)$ & $-0.36(-1.50$ to 0.78$)$ & 0.73 (-0.17 to 1.62$)$ & $0.95(-0.032$ to 1.86$)$ & $-3.25(-6.67$ to 0.17$)$ & $-3.71(-6.26 \text { to }-1.16)^{*}$ \\
\hline 3rd Quartile (6) & 0.14 (-1.05 to 1.33$)$ & $-0.18(-1.63$ to 1.28$)$ & $-0.16(-1.01$ to 0.69$)$ & $-0.11(-0.98$ to 0.75$)$ & $-3.63(-6.75 \text { to }-0.50)^{*}$ & $-2.11(-4.76$ to 0.55$)$ \\
\hline 4th Quartile (7-8) & 2.05 (-0.34 to 4.43$)$ & $2.20(-0.55$ to 4.95$)$ & 0.86 (-0.039 to 1.75$)$ & 0.70 (-0.21 to 1.61$)$ & $-3.00(-6.23$ to 0.23$)$ & 0.26 (-2.38 to 2.91$)$ \\
\hline \multicolumn{7}{|l|}{ Saltpreference } \\
\hline 1stQuartile(16-20) & 1 & 1 & 1 & 1 & 1 & 1 \\
\hline 2nd Quartile (21) & -0.64 (-1.92 to 0.64$)$ & 0.56 (-1.11 to 2.23$)$ & $-0.41(-1.19$ to 0.38$)$ & $-0.69(-1.48$ to 0.11$)$ & 0.75 (-1.92 to 3.42$)$ & $-0.33(-3.48$ to 2.81$)$ \\
\hline 3rd Quartile (22) & -0.13 (-1.78 to 1.52 ) & 0.75 (-1.12 to 2.62$)$ & $-0.29(-1.34$ to 0.76$)$ & $-0.57(-1.62$ to 0.48$)$ & 1.67 (-1.30 to 4.63$)$ & 1.20 (-2.61 to 5.01$)$ \\
\hline 4th Quartile (23-26) & -0.84 (-2.49 to 0.81$)$ & 0.08 (-1.87 to 2.03$)$ & $-1.27(-2.37 \text { to }-0.17)^{*}$ & $-1.36(-2.46 \text { to }-0.26)^{*}$ & 0.50 (-2.17 to 3.17$)$ & 0.16 (-3.88 to 4.19$)$ \\
\hline \multicolumn{7}{|l|}{ Knowledge \&balance } \\
\hline 1stQuartile(4-8) & 1 & 1 & 1 & 1 & 1 & 1 \\
\hline 2nd Quartile (9-10) & 0.68 (-0.64 to 1.99$)$ & $-0.59(-2.20$ to 1.01$)$ & $-0.58(-1.40$ to 0.24$)$ & $-0.32(-1.14$ to 0.50$)$ & $1.00(-1.50$ to 3.50$)$ & $1.72(-0.67$ to 4.12$)$ \\
\hline 3rd Quartile (11) & $0.26(-0.98$ to 1.50$)$ & $0.60(-0.67$ to 1.86$)$ & $-0.62(-1.60$ to 0.37$)$ & $-0.62(-1.59$ to 0.36$)$ & $1.00(-1.82$ to 3.82$)$ & $2.22(-2.08$ to 6.53$)$ \\
\hline 4th Quartile(12-14) & 0.30 (-1.11 to 1.71$)$ & $0.04(-1.56$ to 1.64$)$ & $-0.42(-1.37$ to 0.54$)$ & $-0.08(-1.06$ to 0.90$)$ & $-0.08(-3.10$ to 3.10$)$ & $1.98(-1.83$ to 5.80$)$ \\
\hline \multicolumn{7}{|l|}{ Appetite control } \\
\hline 1stQuartile (4-7) & 1 & 1 & 1 & 1 & 1 & 1 \\
\hline 2nd Quartile (8-9) & -0.91 (-1.86 to 0.046$)$ & $-1.06(-2.09 \text { to }-0.037)^{*}$ & 0.37 (-0.47 to 1.20$)$ & 0.016 ( -0.84 to 0.87 ) & $-0.083(-3.42$ to 3.26$)$ & $-0.34(-4.75$ to 4.06$)$ \\
\hline 3rdQuartile (10-11) & $-2.04(-3.34 \text { to }-0.73)^{*}$ & $-1.54(-3.00 \text { to }-0.096)^{*}$ & 0.55 (-0.28 to 1.38$)$ & 0.64 (-0.18 to 1.46$)$ & $-1.00(-3.68$ to 1.68$)$ & 1.13 (-2.50 to 4.75$)$ \\
\hline 4th Quartile(12-16) & $0.25(-2.80$ to 3.30$)$ & $0.55(-2.80$ to 3.91$)$ & $1.20(-0.0082$ to 2.41$)$ & $1.30(-0.12$ to 2.48$)$ & $0.65(-2.28$ to 3.58$)$ & 1.30 (-2.04 to 4.64$)$ \\
\hline \multicolumn{7}{|l|}{ Eating to food cues } \\
\hline 1stQuartile(16-22) & 1 & 1 & 1 & 1 & 1 & 1 \\
\hline 2nd Quartile (23-25) & $-0.55(-1.74$ to 0.65$)$ & -0.87 (-1.43 to 1.25$)$ & $0.67(-0.19$ to 1.53$)$ & $0.54(-0.32$ to 1.41$)$ & NA & NA \\
\hline 3rd Quartile (26-27) & -0.021 (-1.18 to 1.14) & $-0.16(-1.44$ to 1.11$)$ & $0.037(-0.83$ to 0.90$)$ & $0.10(-0.80$ to 1.00$)$ & 0.40 (-2.85 to 3.65$)$ & 0.68 (-2.91 to 4.28$)$ \\
\hline 4th Quartile (28-35) & $-1.83(-4.26$ to 0.59$)$ & -1.14 (-3.69 to 1.42) & $1.03(0.022 \text { to } 2.04)^{*}$ & 1.09 (0.020 to 2.15$)^{*}$ & $-0.27(-2.64$ to 2.11$)$ & 0.79 (-2.08 to 3.67$)$ \\
\hline
\end{tabular}

Coefficient, confidence interval (low to high) presented for both univariable and multivariable linear regression analysis adjusted for age, alcohol consumption, smoking, medication for diabetes, hypertension, and dyslipidemia, stratified by body mass index (BMI).

*P $<0.05$. 
Table 4. Association between allostatic load index and dietary habits in female subjects (total $\mathrm{n}=58$ )

\begin{tabular}{|c|c|c|c|c|}
\hline \multirow{2}{*}{ Variable } & \multicolumn{2}{|c|}{$\mathrm{BMI}<25$} & \multicolumn{2}{|c|}{$\mathrm{BMI} \geq 25$} \\
\hline & Univariable & Multivariable & Univariable & Multivariable \\
\hline \multicolumn{5}{|l|}{ Regularity } \\
\hline 1st Quartile (4-7) & 1 & 1 & 1 & 1 \\
\hline 2nd Quartile (8-9) & $-0.09(-1.70$ to 1.52$)$ & $0.27(-1.75$ to 2.28$)$ & $-0.54(-2.13$ to 1.07$)$ & $-1.09(-2.42$ to 0.24$)$ \\
\hline 3rd Quartile (10-11) & $0.58(-0.68$ to 1.84$)$ & $0.43(-0.92$ to 1.79$)$ & 0.66 ( -0.83 to 2.15$)$ & 0.04 ( -1.34 to 1.25$)$ \\
\hline 4th Quartile (12-15) & $0.51(-0.78$ to 1.80$)$ & $0.09(-1.49$ to 1.66$)$ & $0.51(-1.05$ to 2.07$)$ & $-1.39(-2.94$ to 0.17$)$ \\
\hline \multicolumn{5}{|l|}{ Carbohydrates preference } \\
\hline 1st Quartile (1-3) & 1 & 1 & 1 & 1 \\
\hline 2nd Quartile (4) & $0.62(-0.70$ to 1.93$)$ & -0.07 ( -1.58 to 1.44$)$ & 0.05 ( -2.05 to 2.15$)$ & -0.73 ( -2.73 to 1.27$)$ \\
\hline 3rd Quartile (5) & 0.25 ( -1.11 to 1.61$)$ & $-0.40(-1.89$ to 1.08$)$ & $0.03(-1.80$ to 1.85$)$ & -0.46 ( -2.06 to 1.14$)$ \\
\hline 4th Quartile (6-8) & 0.08 ( -1.77 to 1.93$)$ & $-0.21(-2.12$ to 1.71$)$ & 0.37 ( -1.79 to 2.53$)$ & -0.53 ( -2.41 to 1.35$)$ \\
\hline \multicolumn{5}{|l|}{ Fat preference } \\
\hline 1st Quartile (2-3) & 1 & 1 & 1 & 1 \\
\hline 2nd Quartile (4) & $-0.45(-2.14$ to 1.23$)$ & -0.24 ( -2.08 to 1.60$)$ & $-1.52(-3.01$ to -0.03$)$ & -0.67 ( -2.01 to 0.68$)$ \\
\hline 3rd Quartile (5-6) & $0.23(-0.91$ to 1.37$)$ & $0.06(-1.20$ to 1.32$)$ & $1.13(-0.30$ to 2.55$)$ & $1.19(-0.055$ to 2.44$)$ \\
\hline 4th Quartile (7-8) & $0.05(-2.24$ to 2.33$)$ & $-0.51(-2.94$ to 1.91$)$ & -0.19 ( -1.48 to 1.09$)$ & -0.48 ( -1.70 to 0.74$)$ \\
\hline \multicolumn{5}{|l|}{ Salt preference } \\
\hline 1st Quartile (18-20) & 1 & 1 & 1 & 1 \\
\hline 2nd Quartile (21) & $-0.22(-1.58$ to 1.14$)$ & $0.25(-1.26$ to 1.76$)$ & -0.41 ( -2.05 to 1.22$)$ & 0.93 ( -0.47 to 2.33$)$ \\
\hline 3rd Quartile (22) & $-0.62(-2.04$ to 0.80$)$ & $-0.21(-1.86$ to 1.44$)$ & -0.85 ( -2.43 to 0.72$)$ & 0.95 ( -0.46 to 2.37$)$ \\
\hline 4th Quartile (23-25) & $-0.10(-1.60$ to 1.40$)$ & -0.59 ( -2.40 to 1.22$)$ & -0.56 ( -2.27 to 1.16$)$ & 0.71 ( -0.81 to 2.23$)$ \\
\hline \multicolumn{5}{|l|}{ Knowledge \& balance } \\
\hline 1st Quartile (4-8) & 1 & 1 & 1 & 1 \\
\hline 2nd Quartile (9) & $0.55(-0.96$ to 2.06$)$ & $1.38(-0.058$ to 2.82$)$ & -0.20 ( -1.58 to 1.18$)$ & -0.11 ( -1.28 to 1.06$)$ \\
\hline 3rd Quartile (10-11) & $0.38(-1.13$ to 1.89$)$ & $1.42(-0.073$ to 2.92$)$ & $0.19(-2.07$ to 2.46$)$ & -0.18 (-1.97 to 1.60$)$ \\
\hline 4th Quartile(12-14) & $0.14(-1.06$ to 1.33$)$ & -0.03 ( -1.16 to 1.09$)$ & -0.70 ( -2.17 to 0.78$)$ & $-1.10(-2.33$ to 0.13$)$ \\
\hline \multicolumn{5}{|l|}{ Appetite control } \\
\hline 1st Quartile (4-8) & 1 & 1 & 1 & 1 \\
\hline 2nd Quartile (9-10) & $0.61(-0.63$ to 1.84$)$ & $0.57(-0.82$ to 1.96$)$ & $0.69(-1.29$ to 2.66$)$ & 0.06 ( -1.93 to 2.06$)$ \\
\hline 3rd Quartile (11-12) & $-0.47(-1.83$ to 0.88$)$ & -0.09 (-1.61 to 1.44$)$ & $1.44(-0.47$ to 3.35$)$ & $0.69(-1.34$ to 2.71$)$ \\
\hline 4th Quartile (13-16) & $0.30(-1.12$ to 1.71$)$ & $0.55(-1.00$ to 2.10$)$ & $1.39(-0.41$ to 3.19$)$ & 0.81 ( -1.12 to 2.74$)$ \\
\hline \multicolumn{5}{|l|}{ Eating to food cues } \\
\hline 1st Quartile (15-22) & 1 & 1 & 1 & 1 \\
\hline 2nd Quartile (23-25) & -0.35 ( -1.49 to 0.79$)$ & -0.17 ( -1.46 to 1.12$)$ & 0.06 (-1.83 to 1.83$)$ & $-0.21(-2.12$ to 1.70$)$ \\
\hline 3rd Quartile (26-28) & 0.08 ( -1.38 to 1.54$)$ & $0.16(-1.49$ to 1.82$)$ & $2.19(0.67 \text { to } 3.71)^{*}$ & $1.40(-0.32$ to 3.12$)$ \\
\hline 4th Quartile (29-58) & $1.08(-0.38$ to 2.54$)$ & 0.76 ( -0.91 to 2.44$)$ & $1.36(-0.194$ to 2.91$)$ & 0.74 ( -1.17 to 2.66$)$ \\
\hline
\end{tabular}

Coefficient, confidence interval (low to high) presented for both univariable and multivariable linear regression analysis adjusted for age, alcohol consumption, smoking, medication for diabetes, hypertension, and dyslipidemia, stratified by body mass index (BMI). $* \mathrm{P}<0.05$. 


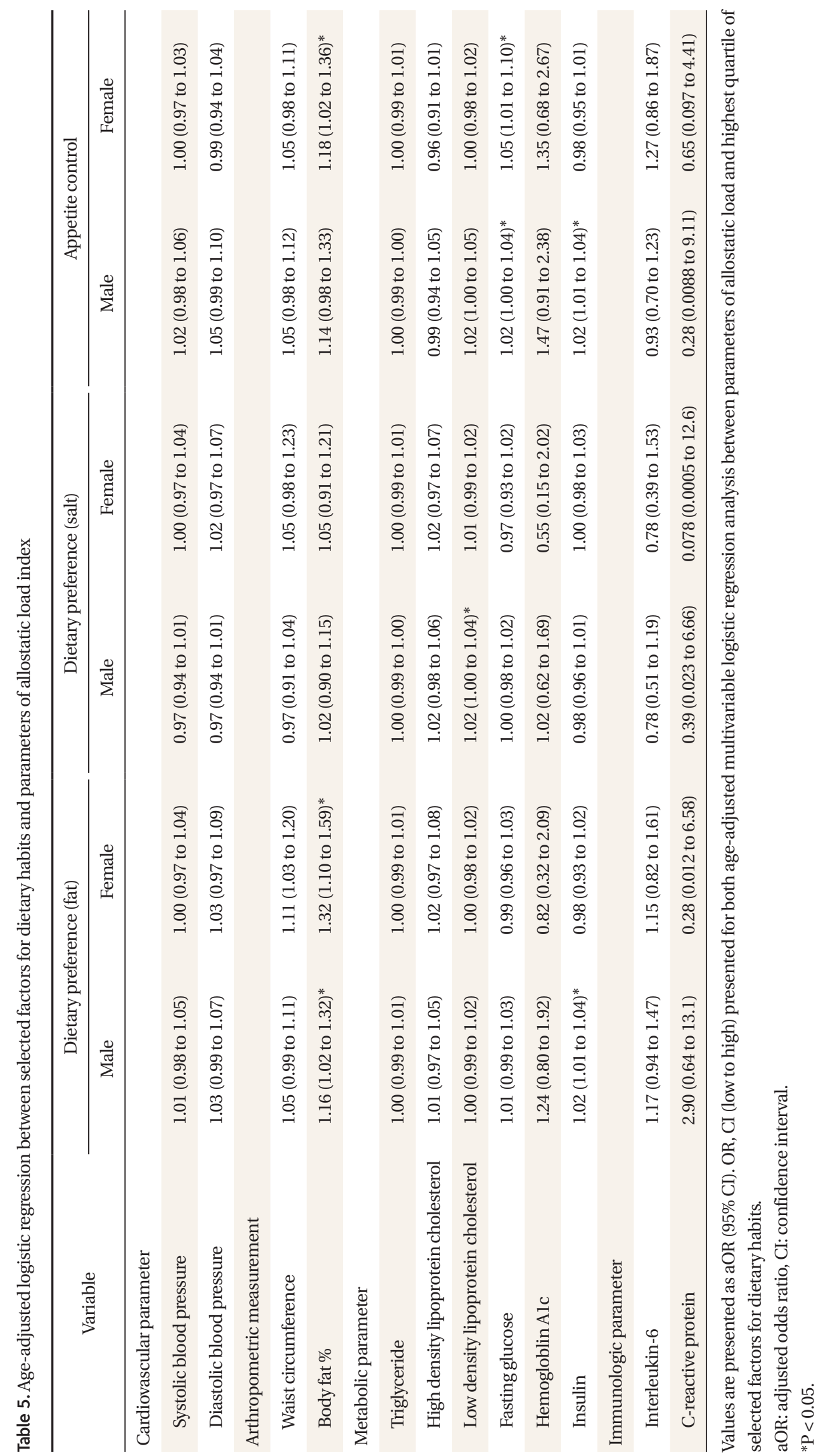



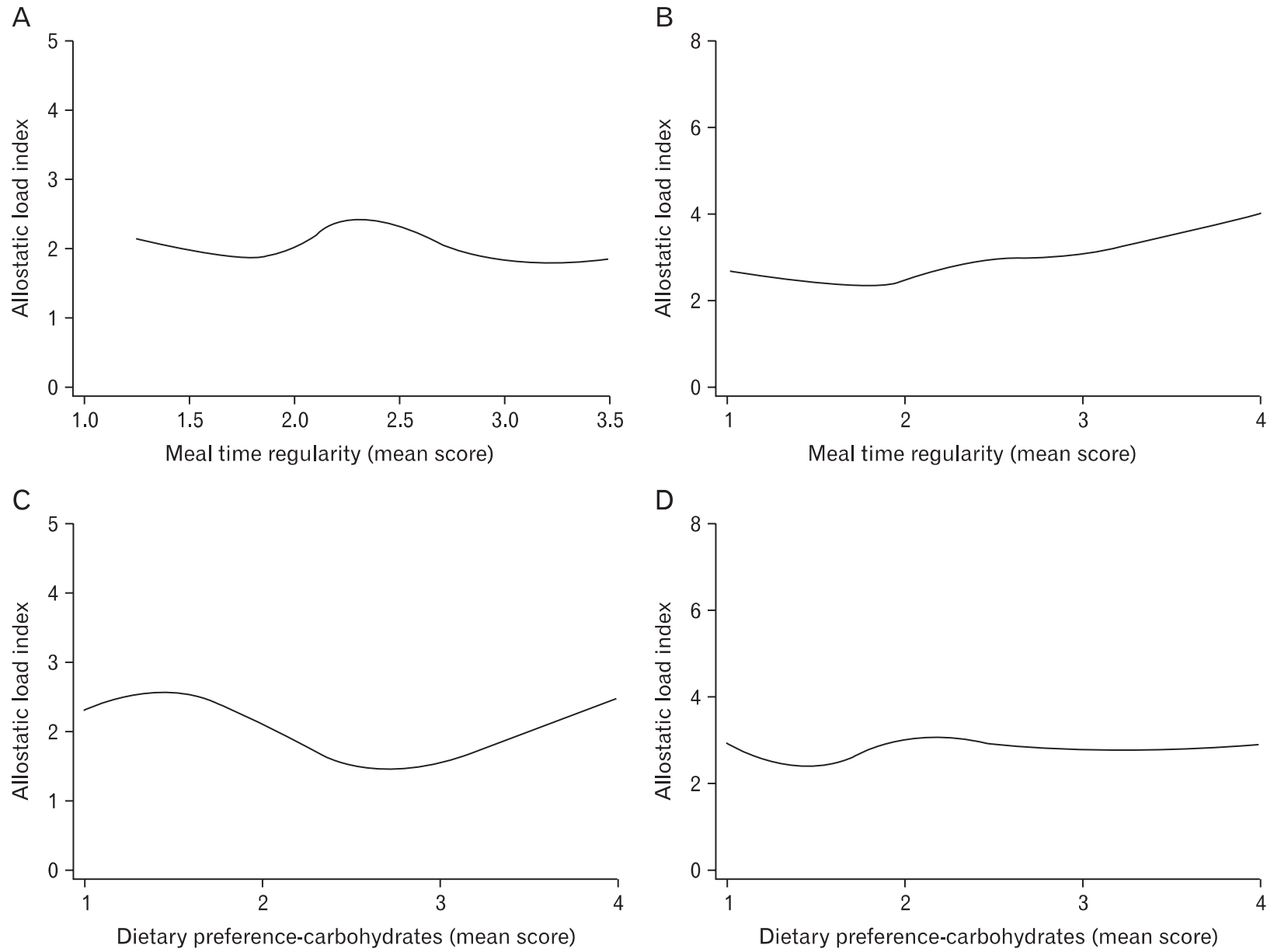

Figure 1. Cubic spline analysis between allostatic load index and dietary habit parameters in non-obese (body mass index $[\mathrm{BMI}]<25$ ) and obese (BMI $\geq 25$ ) male subjects. (A) Meal time regularity in non-obese subjects (BMI < 25). (B) Meal time regularity in obese subjects (BMI $\geq 25$ ). (C) Dietary preference to carbohydrates in non-obese subjects (BMI $<25$ ). (D) Dietary preference to carbohydrates in obese subjects (BMI $\geq 25)$. (E) Dietary preference to fat in non-obese subjects (BMI < 25). (F) Dietary preference to fat in obese subjects (BMI $\geq 25)$. (G) Dietary preference to salt in non-obese subjects (BMI <25). (H) Dietary preference to salt in obese subjects (BMI $\geq 25)$.

\section{Analysis of Trends in Relationship between Allostatic Load and Dietary Habits}

In order to evaluate an overall trend in the relationship between each questionnaire-based dietary habits and AL, cubic spline analysis was performed. Male subjects were stratified into two subgroups-non-obese $(\mathrm{BMI}<25)$ and obese $(\mathrm{BMI}$ $\geq 25$ ), as shown in Figure 1. Meal time irregularity (high mean score) was correlated with higher $\mathrm{AL}$ in obese subjects. No remarkable general trends were observed in dietary preference for carbohydrates. Higher dietary preference for fat was correlated with higher AL in both obese and non-obese subjects. Lower dietary preference for salt was correlated with higher AL, especially in non-obese subjects. Lower knowledge of nutrition and balanced diet was correlated with higher AL only in non- obese patients. Lower appetite control (higher score) was correlated with high AL in both obese and non-obese males, whereas higher appetite control (lower score) was correlated with high $\mathrm{AL}$ in non-obese subjects as in Figure 2. Eating in response to food cues was correlated with high AL.

\section{DISCUSSION}

Since both AL and dietary habits have been associated with chronic conditions and CVD mortality, dietary habits may trigger allostatic overload. Nevertheless there are few studies to investigate this. To our knowledge our study is the first to evaluate various dietary habits in association with allostatic 

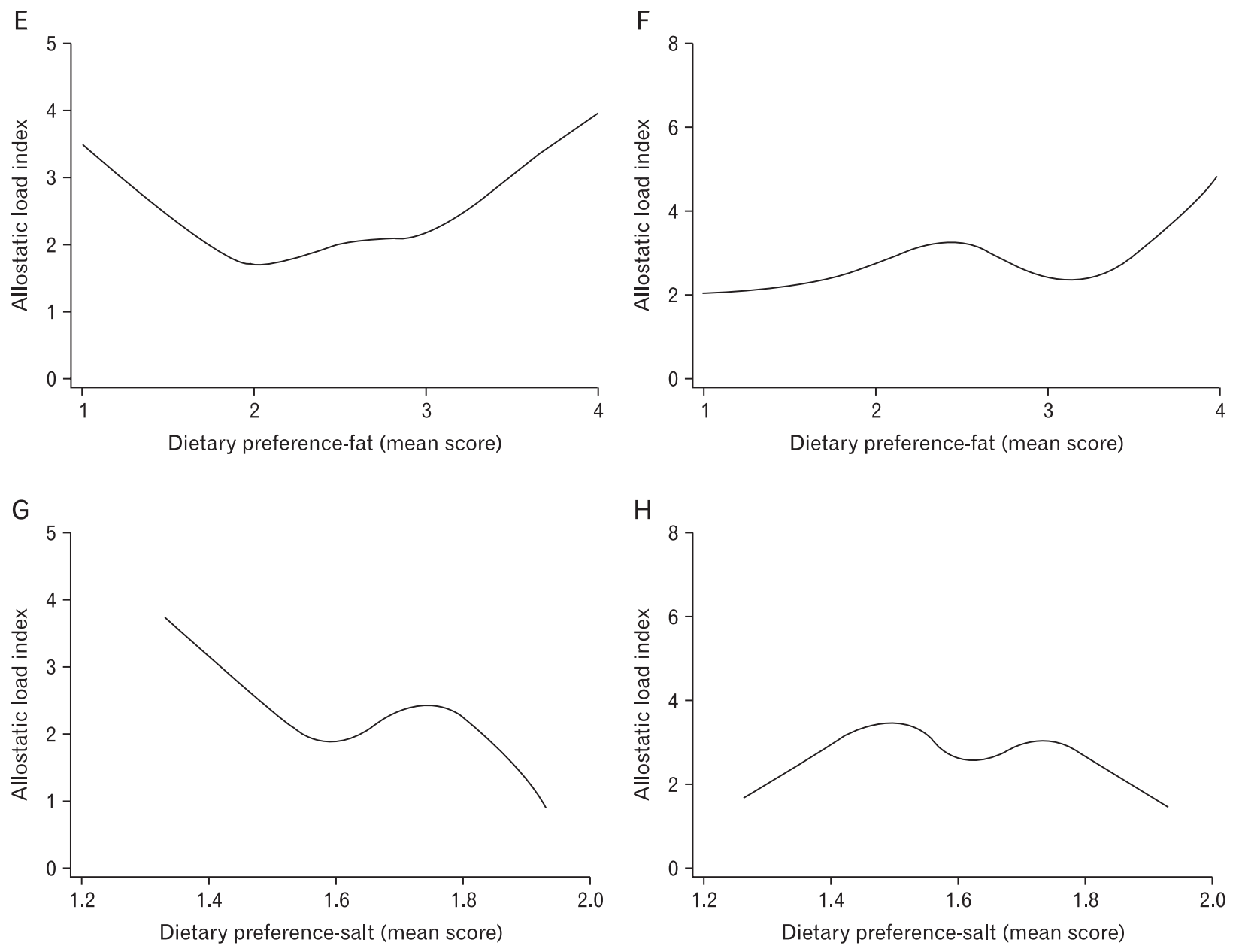

Figure 1. Continued.

load in metabolic syndrome patients. We focused particularly on metabolic syndrome patients, because appropriate lifestyle modification in terms of dietary habits is especially important to this particular population.

Due to insufficient number of subjects, multivariable linear regression analysis of AL did not yield many definitive results, especially in females. Dietary preference for fat, dietary preference for salt, and appetite control are the dietary habit factors that were shown to be significantly associated with $\mathrm{AL}$ in specific subgroups of BMI. Age-adjusted logistic regression analysis between each of the parameters of $\mathrm{AL}$ and the three parameters found to be significantly associated with AL showed significant associations with high insulin, high body fat percentage, and high LDL, but their associations were modest in strength.

The common methods used to determine dose-response and trend analysis for continuous variables, such as linear regression models, can be very misleading because, in essence, they assume that the linear trend curve follows a specific model form. ${ }^{15)}$ This is usually overcome by looking for trends by categorization of variables, but this approach is only adequate if numbers allow the use of categories that reflect homogeneous groups. ${ }^{16)}$ In our study, in order to overcome limited statistical power in our linear regression analysis, we performed cubic spline analysis, a prominent method intermediate between simple regression and nonparametric regression in behavior, ${ }^{17)}$ to observe remarkable trends in the association between AL and questionnaire-based parameters of dietary habits.

Upon cubic spline analysis, meal time irregularity was correlated with higher AL in obese males. Higher dietary preference for fat was correlated with higher AL in both obese and non-obese males. Dietary preference for fat was also associated with higher body fat in both males and females. This result was similar to 
A

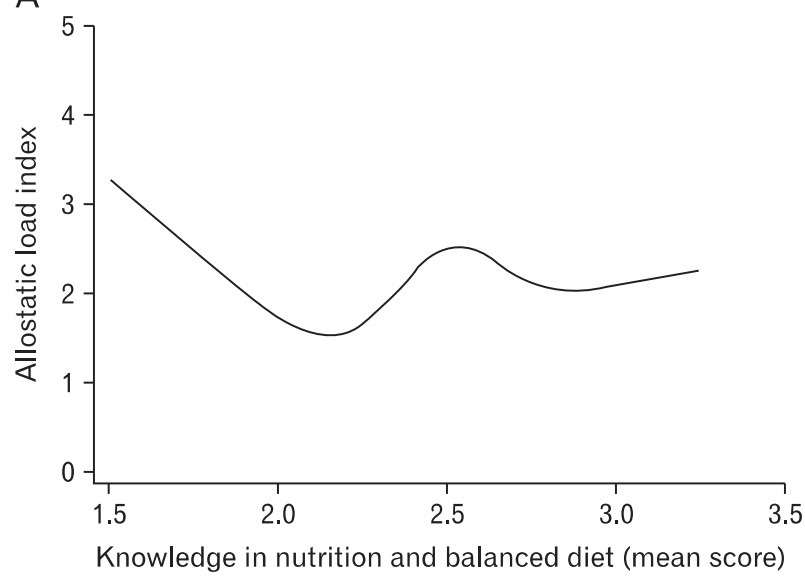

C

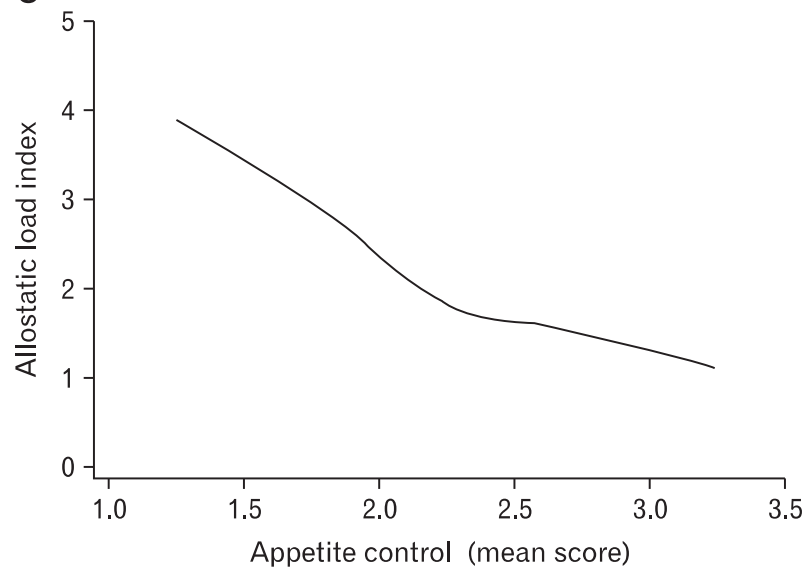

E

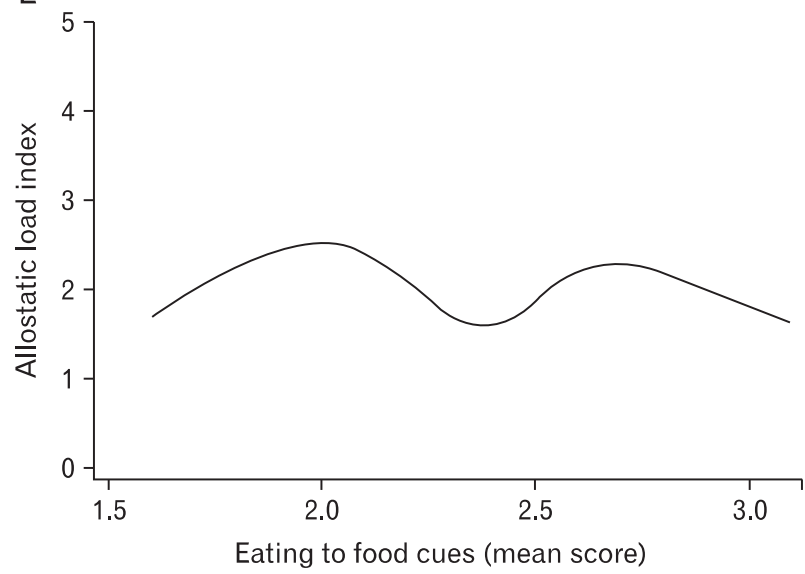

B

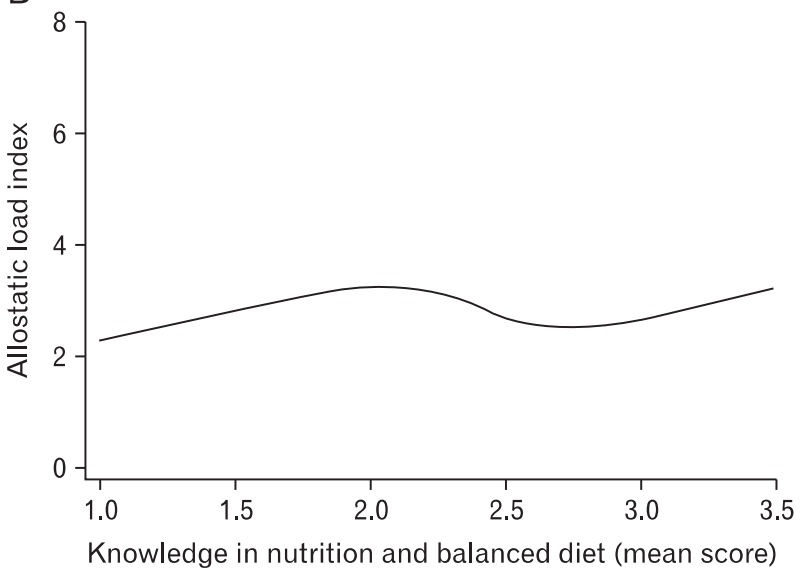

D

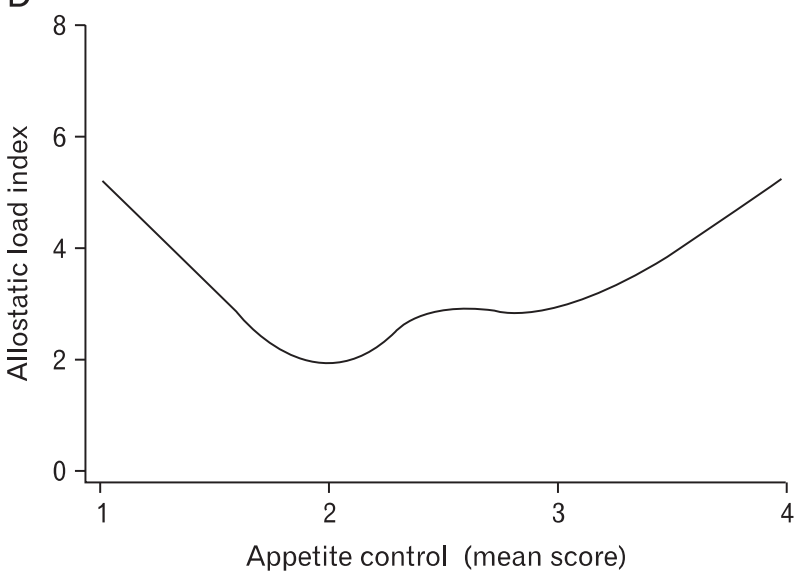

$\mathrm{F}$

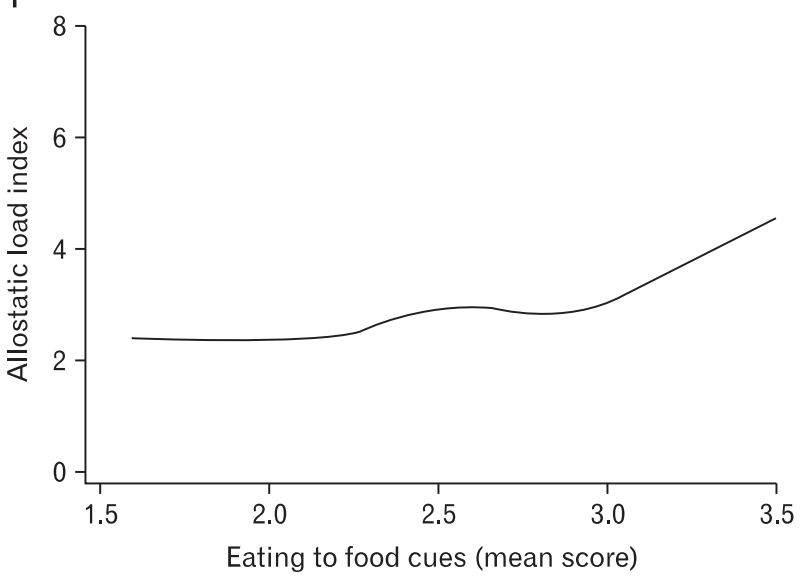

Figure 2. Cubic spline analysis between allostatic load index and dietary habit parameters in non-obese (body mass index [BMI] $<25$ ) and obese (BMI $\geq 25)$ male subjects. (A) Knowledge in nutrition and balanced diet in non-obese subjects (BMI < 25). (B) Knowledge in nutrition and balanced diet in obese subjects (BMI $\geq 25$ ). (C) Appetite control in non-obese subjects (BMI < 25). (D) Appetite control in obese subjects (BMI $\geq 25$ ). (E) Eating to food cues in non-obese subjects (BMI <25). (F) Eating to food cues in obese subjects (BMI $\geq 25$ ).

Mattei's study, ${ }^{11)}$ which suggested that meat, processed meat, and French fries may contribute to allostatic overload in Puerto Rican adults. A number of epidemiological and clinical studies suggest that higher fat intake is associated with type 2 diabetes and insulin resistance. $^{18,19)}$

On the contrary, preference for carbohydrates was not associated 
with higher AL or glucose or insulin level. Further quantitative analysis using food frequent questionnaires with long term data may be useful to confirm a causal relationship between macronutrient intake and health status or AL.

Lower dietary preference for salt was correlated with higher $\mathrm{AL}$, especially in non-obese males. Lower knowledge of nutrition and balanced diet was correlated with higher AL only in nonobese males. Lower appetite control (higher score) was correlated with high AL in both obese and non-obese males, whereas higher appetite control (lower score) was correlated with high $\mathrm{AL}$ in non-obese subjects. Eating in response to food cues was correlated with high AL.

Beyond the modest number of subjects in this study, there are few limitations. This study does not present a casual relationship between AL and dietary habits since it is cross-sectional study. The questionnaire we used to evaluate dietary habits was developed without proper validation, and therefore our results may include systematic biases, and quantitative assessment may be erroneous. Our assessment of dietary habits of subjects is based on responses that are self-reported and subjective in nature, prone to recall bias and subjective discrepancies. The categories in the questionnaire, and the specific items could be generally acceptable since those are used commonly in clinical practice. Also questionnaire items for salt preference were developed with reference to existing research. ${ }^{20)}$

Despite the aforementioned limitations to our study, our study suggests several interesting trends of association between $\mathrm{AL}$ and dietary habits. Due to our use of an unvalidated survey and modest sample number, we cannot make any quantitative conclusions; however, general qualitative implications may be made.

Most of our cubic spline regression analysis results are consistent with the general consensus. Dietary preference for fat, eating in response to food cues, lower knowledge of nutrition and balanced diet were indeed found to be associated to high AL. Our results suggest that excessive appetite control may actually be harmful in terms of AL, possibly due to stress. Surprisingly, lower dietary preference for salt was associated with high $\mathrm{AL}$, possibly suggesting that excess restriction of salt intake may be harmful.

Future studies with a larger sample size and validated surveys with longitudinal data are needed to confirm our results and investigate causality. Additional quantitative measures of actual diet in terms of time of intake, specific amounts in calories, grams of fat, protein, and carbohydrates, and amount of sodium intake, etc., will add confirmatory value.

From a clinical perspective, our results suggest that metabolic syndrome patients, consistent with current guidelines, should be discouraged from eating fat and eating in response to food cues, and should be educated about nutrition and balanced diet. Furthermore, our results suggest excess restriction of salt and excess appetite control may be harmful, reflecting the associated high AL in our study.

\section{CONFLICT OF INTEREST}

No potential conflict of interest relevant to this article was reported.

\section{REFERENCES}

1. McEwen BS, Stellar E. Stress and the individual: mechanisms leading to disease. Arch Intern Med 1993;153:2093-101.

2. Seeman TE, Singer BH, Rowe JW, Horwitz RI, McEwen BS. Price of adaptation: allostatic load and its health consequences. MacArthur studies of successful aging. Arch Intern Med 1997;157:2259-68.

3. Karlamangla AS, Singer BH, McEwen BS, Rowe JW, Seeman TE. Allostatic load as a predictor of functional decline. MacArthur studies of successful aging. J Clin Epidemiol 2002;55:696-710.

4. Seeman TE, McEwen BS, Rowe JW, Singer BH. Allostatic load as a marker of cumulative biological risk: MacArthur studies of successful aging. Proc Natl Acad Sci U S A 2001;98: 4770-5.

5. McEwen BS. Protective and damaging effects of stress mediators. N Engl J Med 1998;338:171-9.

6. McEwen BS, Seeman T. Protective and damaging effects of mediators of stress: elaborating and testing the concepts of allostasis and allostatic load. Ann N Y Acad Sci 1999;896:3047.

7. Paradis AM, Godin G, Perusse L, Vohl MC. Associations between dietary patterns and obesity phenotypes. Int J Obes 
(Lond) 2009;33:1419-26.

8. Heidemann C, Schulze MB, Franco OH, van Dam RM, Mantzoros CS, Hu FB. Dietary patterns and risk of mortality from cardiovascular disease, cancer, and all causes in a prospective cohort of women. Circulation 2008;118:230-7.

9. McEwen BS. Stress, adaptation, and disease: allostasis and allostatic load. Ann N Y Acad Sci 1998;840:33-44.

10. McEwen BS, Wingfield JC. The concept of allostasis in biology and biomedicine. Horm Behav 2003;43:2-15.

11. Mattei J, Noel SE, Tucker KL. A meat, processed meat, and French fries dietary pattern is associated with high allostatic load in Puerto Rican older adults. J Am Diet Assoc 2011;111: 1498-506.

12. Mattei J, Demissie S, Falcon LM, Ordovas JM, Tucker K. Allostatic load is associated with chronic conditions in the Boston Puerto Rican Health Study. Soc Sci Med 2010;70:1988-96.

13. Allsworth JE, Weitzen S, Boardman LA. Early age at menarche and allostatic load: data from the Third National Health and Nutrition Examination Survey. Ann Epidemiol 2005; 15:43844.
14. Juster RP, McEwen BS, Lupien SJ. Allostatic load biomarkers of chronic stress and impact on health and cognition. Neurosci Biobehav Rev 2010;35:2-16.

15. Maclure M, Greenland S. Tests for trend and dose response: misinterpretations and alternatives. Am J Epidemiol 1992; 135:96-104.

16. Greenland S. Avoiding power loss associated with categorization and ordinal scores in dose-response and trend analysis. Epidemiology 1995;6:450-4.

17. Greenland S. Dose-response and trend analysis in epidemiology: alternatives to categorical analysis. Epidemiology 1995;6:35665.

18. Lovejoy JC. The influence of dietary fat on insulin resistance. Curr Diab Rep 2002;2:435-40.

19. van Dam RM, Willett WC, Rimm EB, Stampfer MJ, Hu FB. Dietary fat and meat intake in relation to risk of type 2 diabetes in men. Diabetes Care 2002;25:417-24.

20. Son SM. Pilot study for low salt consumption projects for Korean people. Seoul: Ministry of Health and Welfare; 2009. 